\title{
GI-MS45-02 | SeCRETS to A Successful Collaboration
}

Moraes, Isabel (National Physical Laboratory, Teddington, GBR)

In the world where multidisciplinary approaches are crucial to achieve a high impact scientific success, effective collaborations are a must!

Collaborative effectiveness is always a complex process that depends of many variables such as interpersonal skills, trust, culture, and occasionally of the physical distance between collaborators. In this interactive talk, approaches to a successful collaboration will be discussed. We must always remember that scientific collaborations are based on passion for a relevant scientific subject! 